Vivian Palmieri

\title{
Calibração do Modelo Qual2E para o Rio Corumbataí (SP)
}

\author{
DissertaÇÃo de MESTRAdo
}

Deparatmento de CiênCia dos Materias e

MetaluRgia

Programa de Pós-Graduação em Engenharia

Metalúrgica

Rio de Janeiro

Agosto de 2003 


\section{Pontifíaia U Uiversidade $C_{\text {atólica }}$

Vivian Palmieri

\section{Calibração do Modelo Qual2E para o Rio Corumbataí (SP)}

\section{Dissertação de Mestrado}

Dissertação apresentada ao Programa de PósGraduação em Engenharia Metalúrgica da PUC-Rio como requisito parcial para obtenção do título de Mestre em Engenharia Metalúrgica.

Orientador: Prof. Roberto José de Carvalho 


\title{
Pontifíla $U_{\text {Niversidade }} C_{\text {attólica }}$

Vivian Palmieri

\section{Calibração do Modelo Qual2E para o Rio Corumbataí (SP)}

\begin{abstract}
Dissertação apresentada como requisito parcial para obtenção do grau de Mestre pelo Programa de PósGraduação em Engenharia Metalúrgica da PUC-Rio. Aprovada pela Comissão Examinadora abaixo assinada.
\end{abstract}

Prof. Roberto José de Carvalho

Orientador

Departamento de Ciência dos Materiais e Metalurgia, PUC-Rio

Prof. Antônio José da Silva Neto

Instituto Politécnico - Universidade do Estado do Rio de Janeiro

Prof. Maurício Leonardo Torem

Departamento de Ciência dos Materiais e Metalurgia, PUC-Rio

Prof. Ney Augusto Dumont

Coordenador Setorial do Centro Técnico Científico, PUC-Rio

Rio de Janeiro, 29 de Agosto de 2003. 
Todos os direitos reservados. É proibida a reprodução total ou parcial do trabalho sem autorização da universidade, da autora e do orientador.

\section{Vivian Palmieri}

Graduou-se em Engenharia Química pela UFRJ (Universidade Federal do Rio de Janeiro) em 1999. Durante a graduação, desenvolveu projetos de iniciação científica em centros conceituados de pesquisas como CENPES, CETEM e COPPE. Durante o curso de mestrado na PUC- Rio (DCMM), elaborou e apresentou trabalhos sobre tecnologia ambiental na indústria química em seminaries. Publicou recentemente em revista especializada. Atualmente, cursa MBA em Marketing pela FGV - Campinas e atua como Engenheira de Produtos na Votorantim Celulose e Papel, unidade de Piracicaba - SP.

Ficha Catalográfica

Palmieri, Vivian

Calibração do modelo Qual2E para o rio Corumbataí(SP) / Vivian Palmieri; orientador: Roberto José de Carvalho. Rio de Janeiro : PUC, Departamento de Engenharia Metalúrgica, 2003.

$91 \mathrm{f}$ : : il. ; $30 \mathrm{~cm}$

Dissertação (mestrado) - Pontifícia Universidade Católica do Rio de Janeiro, Departamento de Engenharia Metalúrgica.

Inclui referências bibliográficas.

1. Engenharia metalúrgica - Teses. 2. Modelo Qual2E. 3. Rio Corumbataí. 4. Calibração. 5. Recursos hídricos. 6. Bacia hidrográfica. I. Carvalho, Roberto José de. II. Pontifícia Universidade Católica do Rio de Janeiro. Departamento de Engenharia Metalúrgica. III. Título.

CDD: 669 


\section{AGRADECIMENTOS}

Ao meu grande amigo e mentor, DSc. Evandro Gaiad Fischer, da CETESB Piracicaba, cuja participação foi fundamental para a elaboração deste trabalho; por sua paciência, confiança e generosidade; pelo conhecimento e vivência transmitidos.

Ao meu orientador, Prof. Roberto de Carvalho, pelo apoio, amizade, estímulo e confiança concedidos, sem os quais a jornada teria sido muito mais difícil, ou mesmo impossível.

À minha mãe, pela eterna confiança depositada nos momentos de dúvida e pelo amor de sempre.

A todos os meus amigos da PUC-Rio, em especial à Adriana e Bruno, pela amizade e generosidade gratuitas, além de todo o apoio logístico prestado em função da distância física.

Aos professores Maurício Torem, Francisco Moura e Eduardo Brocchi, pela confiança e por não permitirem que a distância me impedisse de alcançar o objetivo traçado.

Aos queridos amigos e funcionários do DCMM, Luzinete, Amarildo e Celina, cuja boa vontade foi sempre inquestionável.

Aos meus amigos de infância Marcos, Cibele, Luciana e Cristiane, pelo carinho, admiração e respeito pelas minhas decisões pessoais e profissionais.

A Deus, por mais esta vitória concedida. 


\section{RESUMO}

Palmieri, Vivian; Carvalho, Roberto. CALIBRAÇÃO DO MODELO QUAL2E

PARA O RIO CORUMBATAí (SP). Rio de Janeiro, 2003. 81p. Dissertação de Mestrado - Departamento de Ciência dos Materiais e Metalurgia, Pontifícia Universidade Católica do Rio de Janeiro.

A previsão dos efeitos poluidores sobre uma bacia hidrográfica é uma constante necessidade para os órgãos de gestão ambiental e para os tomadores de decisão neste âmbito. Dentro deste contexto, os modelos matemáticos que permitam prever danos ou melhorias na qualidade de água de bacias hidrográficas são importantes ferramentas para este fim. O Qual2E desponta como uma destas ferramentas, de acesso livre, aplicável à sistemas fluviais, unidimensionais, bem misturados e de fluxo constante. Este trabalho propõe uma aplicação do Qual2E para o rio Corumbataí, localizado no interior do Estado de São Paulo, visando a obtenção de uma curva representativa para a qualidade de água do rio. Para tal, foi utilizada a metodologia sugerida pelo modelo, que prevê a divisão do sistema em trechos de características hidráulicas semelhantes e a subdivisão desses trechos em elementos de mesma extensão - os elementos computacionais (ECs). Os dados de vazão, profundidade, concentração de OD (oxigênio dissolvido), DBO (demanda bioquímica de oxigênio), temperatura, carga e localização de fontes poluidoras foram obtidos através de um projeto de parceria da CETESB com a USP, que contempla a elaboração de um banco de dados para qualidade de água da bacia. Este banco, georreferenciado, foi utilizado tanto para a entrada de condições iniciais no modelo, como para a construção da curva de referência dos dados reais de campo, para comparação com a curva calculada pelo modelo. Os coeficientes de reação resultantes da interação entre as variáveis OD e DBO - presentes no sistema de equações diferenciais resolvidas pelo modelo, foram estimados através de tentativa e erro, até a concordância com os dados observados. A calibração efetuada se mostrou eficiente em reproduzir os dados observados, validando os parâmetros estimados através de um outro conjunto de dados. Uma análise de sensibilidade foi realizada para os parâmetros $\mathrm{k}_{1}, \mathrm{k}_{2}, \mathrm{k}_{3}$ e $\mathrm{k}_{4}$ e a curva calculada foi mais sensível ao coeficiente de decaimento de DBO. As limitações inerentes ao modelo, à coleta de dados e ao tratamento estatístico dos dados disponíveis impediram uma melhor concordância da curva calculada com a observada.

Palavras-chave: modelo Qual2E; rio Corumbataí; calibração; recursos hídricos; bacia hidrográfica. 


\section{ABSTRACT}

Palmieri, Vivian; Carvalho, Roberto. CALIBRATION OF QUAL2E MODEL

FOR CORUMBATAÍ RIVER (SP). Rio de Janeiro, 2003. 81p. Dissertação de Mestrado - Departamento de Ciência dos Materiais e Metalurgia, Pontifícia Universidade Católica do Rio de Janeiro.

The forecast of the polluting effects over a hydric basin is a constant need for the official environmental managers and for the people responsible for the decisions in this subject. Therefore, mathematical models that allow forecast damages or improvements in the river basin water quality are important tools for this purpose. Qual2E appears as one of these tools, of free access, and suitable for one-dimensional well-mixed rivers with constant flow. The objective of this work is to apply the Qual2E model to Corumbataí River, located in São Paulo State, in order to obtain a representative curve of the river water quality. The method suggested by the model request the division of the river in reaches with similar hydric features and a subdivision of these reaches in computational elements (CEs) with the same extension. The flow data, depth, DO (Dissolved Oxygen) concentration, BOD (Biochemical Oxygen Demand), temperature, load and location of pollution sources were obtained from a joint project between CETESB and USP, that resulted in a database for the water quality of the basin. This geo-referenced database was used both for the initial conditions to the model, as for the determination of a reference curve with the real field data, for comparison with the calculated curve of the model. The reaction coefficients - resulting from the interaction between DO and BOD - are the constants of the differential equations system solved by the model, were estimated through trial and error, until the agreement with the observed data. The calibration was efficient to reproduce the field data, through the validation for another group of data. A sensitivity analysis was executed for the parameters $\mathrm{k}_{1}, \mathrm{k}_{2}, \mathrm{k}_{3}$ and $\mathrm{k}_{4}$ and the calculated curve seemed to be more sensible to the BOD decaying coefficient. The limitations inherent to the model, the data collecting and the statistical treatment of the available data did not allow a better agreement between the calculated and the observed curves.

Keywords: Qual2E model; Corumbataí River; calibration; hydric resources; hydrographic basin 


\section{SUMÁRIO}

\section{INTRODUÇÃO}

$\begin{array}{ll}\text { 1.1. Objetivo do Trabalho } & 17\end{array}$

1.2. Introdução à Gestão dos Recursos Hídricos 18

1.3. Descrição da Região em Estudo 19

$\begin{array}{ll}\text { 1.3.1. Panorama físico } & 19\end{array}$

1.3.2. Panorama Sócio-Econômico 23

1.3.3. Situação atual de Gerenciamento da Bacia 25

2. REVISÃO DA LITERATURA

$\begin{array}{ll}\text { 2.1. Trabalhos relacionados } & 27\end{array}$

2.2. Limitações do modelo 30

2.2.1. Na aplicação 31

2.2.2. Na formulação matemática 31

2.2.3. Na calibração $\quad 32$

2.2.4. Na coleta dos dados 33

3. DESCRIÇÃO DO MODELO

3.1.Introdução

3.2.Histórico do modelo $\quad 35$

3.3.Representação conceitual 36 
3.4.1. Equação de transporte de massa

3.4.2. Características hidráulicas

3.4.3. Dispersão longitudinal

3.5.Inter-relações entre parâmetros - OD e DBO

3.5.1. Conceitos

3.5.2. Abordagem matemática dos parâmetros de qualidade

3.5.3. Estimativa do coeficiente de reaeração $\mathrm{k}_{2}$

3.5.4. Barragens

4. METODOLOGIA APLICADA

4.1.Levantamento dos dados

4.2.Discretização do sistema

4.3.Localização de cargas, retiradas e barragens

4.4.Coeficientes hidráulicos

4.5.Coeficientes de reação

4.6.Condições iniciais

4.7.Cargas pontuais e retiradas

4.8.Algoritmo de calibração

66

4.9. Validação da calibração 
5. DISCUSSÃO DOS RESULTADOS

5.1.Calibração do modelo

5.2. Validação do modelo

6. CONCLUSÕES

79

7. SUGESTÕES E PRÓXIMOS PASSOS

83

8. REFERÊNCIAS

85 


\section{LISTA DE FIGURAS}

Figura 1: Localização da Bacia do Corumbataí no Estado de São Paulo.

Figura 2: Bacia do Corumbataí detalhada em seu curso principal e tributários.

Figura 3: Perfil topográfico longitudinal do Rio Corumbataí da nascente à foz.

Figura 4: Fotografias de pontos da bacia do Corumbataí: (a) Nascente em Analândia; (b) Barragem em Rio Claro; (c) Confluência do Ribeirão Claro com o Corumbataí.

Figura 5: Distribuição dos recursos hídricos na bacia do Corumbataí.

Figura 6: Qualidade atual da águas do rio Corumbataí.

Figura 7: Representação esquemática da subdivisão de um trecho de rio, segundo metodologia do Qual2E.

Figura 8: Principais interações entre parâmetros de qualidade no Qual2E.

Figura 9: Tela do Qual2E - divisão do sistema em trechos - e esquema da metodologia do modelo.

Figura 10: Representação esquemática da discretização da bacia do Corumbataí.

Figura 11: Representação detalhada dos trechos em ECs e localização das fontes externas.

Figura 12: Esquema unifilar da Bacia do Corumbataí - industrias, municípios e tributários.

Figura 13: Variação do perfil do ponto nascente em Analândia

Figura 15: Entrada de coeficientes hidráulicos no Qual2E. 
Figura 16: Condições de contorno para a calibração.

Figura 17: Cargas pontuais e retiradas no Corumbataí para calibração do modelo.

Figura 18: Algoritmo de cálculo utilizado pelo Qual2E.

Figura 19: Cargas pontuais e retiradas para validação do modelo.

Figura 20: Calibração do modelo para o Rio Corumbataí - OD;

Figura 21: Calibração do modelo para o Rio Corumbataí - DBO.

Figura 22: Sensibilidade do modelo quanto ao coeficiente $\mathrm{K}_{3}$ na curva gerada pelo modelo.

Figura 23: Sensibilidade do modelo quanto ao coeficiente $\mathrm{K}_{4}$ na curva gerada pelo modelo

Figura 24: Sensibilidade do modelo quanto ao coeficiente de reaeração $\mathrm{K}_{2}$ : (a) Owens \& Gibbs; (b) O’Connor \& Dobbins; (c) Churchill.

Figura 25: Resultado da calibração para o tributário Passa Cinco - curvas de OD e DBO.

Figura 26: Validação do modelo para OD -Cenário: ano de 2002.

Figura 27 Validação do modelo para DBO -Cenário: ano de 2002. 


\section{LISTA DE TABELAS}

Tabela 1: Valores experimentais para $\mathrm{D}_{\mathrm{L}}$ encontrados na literatura.

Tabela 2: Exata localização dos trechos do Rio Corumbataí, utilizados na metodologia sugerida pelo modelo.

Tabela 3: Limites de referência dos valores observados (média \pm desvio padrão).

Tabela 4: Coeficientes de reação sugeridos pelo Qual2E.

Tabela 5: Coeficientes da calibração do modelo.

Tabela 6: Agrupamento de fonte pontuais como um só lançamento na foz dos tributários Servidão e Ribeirão Claro.

Tabela 7: Principais alterações de cargas de poluentes entre os anos de 1999 (calibração) e 2002 (validação). 


\section{LISTA DE SÍMBOLOS}

$\alpha_{3}$ é a taxa de oxigênio produzido por fotossíntese, por unidade de alga (mg-O/mgA);

$\alpha_{4}$ é a taxa de remoção de oxigênio por unidade de alga respirada (mg-O/mg-A);

$\alpha_{5}$ é a taxa de remoção de oxigênio por unidade de oxidação de amônia (mg-O/mg$\mathrm{N})$;

$\alpha_{6}$ é a taxa de remoção de oxigênio por unidade de oxidação de nitrito (mg-O/mg-N);

$\mathrm{A}_{\mathrm{x}}$ é a área da seção transversal $\left(\mathrm{L}^{2}\right)$;

A é a concentração de biomassa algal (mg-A/L);

$a, b$ são constantes hidráulicas empíricas (adimensionais);

a é um fator empírico de qualidade de água (adimensional);

b é um coeficiente empírico de aeração por barragem (adimensional);

$\beta_{1}$ é o coeficiente cinético da oxidação de amônia, dependente da temperatura $\left(\mathrm{T}^{-1}\right)$;

$\beta_{2}$ é o coeficiente cinético da oxidação de nitrito, dependente da temperatura $\left(\mathrm{T}^{-1}\right)$;

$c$ é o coeficiente de escape $\left(\mathrm{ft}^{-1}\right)$;

C é a concentração $\left(M . L^{-3}\right)$;

CDBO é a demanda bioquímica carbonácea de oxigênio (mg/L);

DBO é a demanda bioquímica de oxigênio (mg/L); 
$\mathrm{DBO}_{5}$ é a DBO medida após 5 dias de teste $(\mathrm{mg} / \mathrm{L})$;

$\mathrm{DBO}_{20}$ é a DBO medida após 20 dias, valor total (mg/L);

$\mathrm{D}_{\mathrm{a}}$ é o deficit de $\mathrm{O}_{2}$ acima da barragem, $\mathrm{mg} / \mathrm{L}$;

$\mathrm{D}_{\mathrm{b}}$ é o deficit de $\mathrm{O}_{2}$ abaixo da barragem, mg/L;

$\mathrm{D}_{\mathrm{L}}$ é o coeficiente de dispersão longitudinal $\left(\mathrm{L}^{2} \mathrm{~T}^{-1}\right)$;

DSO é a demanda sedimentar de oxigênio (mg/L);

F é o número de Froude (adimensional);

g é a aceleração da gravidade $\left(\mathrm{m} / \mathrm{s}^{2}\right)$;

H é a altura da queda d'água (L);

$\Delta \mathrm{h}$ é a variação na elevação da superfície no trecho (ft);

Lé a DBO ou concentração de substrato do sistema $\left(\mathrm{M} / \mathrm{L}^{3}\right)$;

$\mathrm{k}_{1}$ é o coeficiente de desoxigenação, dependente da temperatura $(1 / \mathrm{T})$;

$\mathrm{k}_{2}$ é o coeficiente de reaeração, dependente da temperatura (1/T);

$\mathrm{k}_{3}$ é a taxa de perda de DBO devido à sedimentação, dependente da temperatura $(1 / \mathrm{T})$

$\mathrm{k}_{4}$ é o coeficiente de demanda bentônica de oxigênio, dependente da temperatura $\left(\mathrm{M} / \mathrm{L}^{2} \cdot \mathrm{T}\right)$;

K é a constante de dispersão (adimensional);

K

$\mu$ é a taxa de crescimento de algas, dependente da temperatura $\left(\mathrm{T}^{-1}\right)$;

M é a massa (M); 
n é o fator de rugosidade de Manning (adimensional);

$\mathrm{N}_{1}$ é a concentração de amônia (mg-N/L);

$\mathrm{N}_{2}$ é a concentração de nitrito (mg-N/L);

O é a concentração de $\mathrm{OD}\left(\mathrm{M} / \mathrm{L}^{3}\right)$;

O* é a concentração de saturação de oxigênio em água, a dada temperatura $\left(\mathrm{M} / \mathrm{L}^{3}\right)$;

$\rho$ é a taxa de respiração das algas, dependente da temperatura $\left(\mathrm{T}^{-1}\right)$;

$Q$ é a vazão ou fluxo no trecho $\left(\mathrm{L}^{3}\right)$;

$\mathrm{R}$ é o raio hidráulico $(\mathrm{L})$;

s é a fonte ou sumidouro externo (M.T ${ }^{1}$ ).

Se é a inclinação da reta do gradiente de energia (adimensional);

$S_{\mathrm{o}}$ é a inclinação do leito (adimensional);

t é o tempo (T);

$T$ é a temperatura $\left({ }^{\circ} \mathrm{C}\right)$;

$\bar{u}$ é a velocidade média $\left(\mathrm{L} . \mathrm{T}^{-1}\right)$;

$\mathrm{u}^{*}$ é a velocidade média de cisalhamento $\left(\mathrm{L} . \mathrm{T}^{-1}\right)$;

$\mathrm{V}=\mathrm{A}_{\mathrm{x}} \mathrm{dx}$ é o incremento de volume $\left(\mathrm{L}^{3}\right)$;

x é a distância (L). 
"Não haverá, para os sistemas aniquilados

Dia seguinte.

O ranúculo da esperança não brota

No dia seguinte.

A vida harmoniosa não se restaura

No dia seguinte.

O vazio da noite, o vazio de tudo

Será o dia seguinte."

Carlos Drummond de Andrade 